\title{
Linx
}

Revue des linguistes de l'université Paris X Nanterre

39 | 1998

Modèles linguistiques : convergences, divergences

\section{QUI ? Un pronom indéfini}

Qui? an indefinite pronoun

\section{Annie Delaveau}

\section{OpenEdition}

Journals

Édition électronique

URL : http://journals.openedition.org/linx/877

DOI : 10.4000/linx.877

ISSN : 2118-9692

\section{Éditeur}

Presses universitaires de Paris Nanterre

\section{Édition imprimée}

Date de publication : 15 décembre 1998

Pagination : 71-87

ISSN : 0246-8743

Référence électronique

Annie Delaveau, « QUI ? Un pronom indéfini », Linx [En ligne], 39 | 1998, mis en ligne le 02 juillet 2012, consulté le 19 avril 2019. URL : http://journals.openedition.org/linx/877 ; DOI : 10.4000/linx.877

Ce document a été généré automatiquement le 19 avril 2019

Département de Sciences du langage, Université Paris Ouest 


\title{
QUI ? Un pronom indéfini
}

\author{
Qui? an indefinite pronoun
}

\section{Annie Delaveau}

Dans les grammaires en usage, le pronom qui est traité comme une unité qui appartient à la catégorie des pronoms interrogatifs ; par ce traitement qui interrogatif est isolé, ce qui interdit de voir que le même mot qui a d'autres emplois, puisque les rapprochements qui permettraient d'unifier les emplois de ce mot ne sont pas faits : il s'agit du qui distributif (1), du qui relatif sans antécédent (2), du qui des indéterminatives généralisées ${ }^{1}$ (3), et enfin du qui, propre au relatif prépositionnel (4) :

1. Chaque habitué apportait qui sa canne, qui son manteau, qui sa lanterne. (Balzac, La Vieille fille)

2. Qui m'aime me suive.

3. Qui que ce soit qui vienne, on sera content.

4. L'homme à qui je parle.

2 Dire que toutes ces occurrences sont celles d'un seul mot suppose quelque raisonnement et quelques hypothèses. En effet, les mots grammaticaux sont ordinairement organisés en classes sur la base d'une propriété qui permet de les regrouper avec une série de même fonctionnement. La ressemblance se manifeste dans des tableaux qui rassemblent dans un espace à deux dimensions des formes qui ont en commun la catégorie qui donne un titre au tableau : pronoms personnels, pronoms interrogatifs, pronoms relatifs.

3 La perspective développée ici est toute différente : il s'agit non d'envisager un mot dans les relations qu'il entretient avec d'autres mots de même appartenance, mais dans la relation qu'il entretient avec lui-même à travers des emplois, dont il faut démontrer qu'ils exhibent bien le même mot. Cette démonstration se fait par la confrontation non seulement des variations de distribution et d'interprétation du mot, mais aussi d'autres combinatoires qui mettent en jeu des catégories autres. 
Cette perspective de traitement des mots grammaticaux s'appuie sur une proposition de Emonds (1985) qui énonce ce qu'on peut appeler «Principe de singularité des mots grammaticaux » :

« Aucun mot grammatical ne diffère d'aucun autre uniquement par des propriétés sémantiques.» (p.165)

Ce principe, que Emonds nomme « Principe de comportement syntaxique unique (Unique Syntactic Behavior) » peut être interprété comme signifiant que chaque mot grammatical diffère de tout autre appartenant à la même liste par au moins une propriété combinatoire. Il y a donc une propriété syntaxique, à savoir au moins une distribution singulière, qui est propre à chaque mot grammatical.

Ceci constitue une des différences importantes entre les mots grammaticaux et les mots lexicaux, puisque pour ces derniers on peut former des classes telles que tous les éléments de la classe partagent ensemble un grand nombre de propriétés formelles et ne diffèrent entre eux que par le sens qui les définit: ainsi tous les noms entrent dans le contexte d'un déterminant à leur gauche. On peut donc avancer qu'un des caractères des mots grammaticaux consiste dans leur capacité à entrer dans des contextes et à en refuser d'autres d'une façon qui est pour chacun singulière.

7 Cette singularité des mots grammaticaux a une conséquence: c'est qu'aucune terminologie ne leur convient, puisque les terminologies utilisées couramment pour désigner un mot grammatical consistent à privilégier une distribution, à l'exclusion d'autres qui particularisent chaque mot grammatical, et ces terminologies conduisent à camoufler cette singularité qui se manifeste justement par l'appartenance du mot grammatical à plusieurs listes, plusieurs tableaux.

Du coup l'identité singulière de chaque mot est effacée au profit d'un éclatement qui ne permet pas toujours de constituer ce que j'appelle la carte d'identité du mot ; cela conduit en même temps souvent à construire l'idée qu'il y a des homonymes, là où il y a un seul mot.

9 Construire une carte d'identité des mots grammaticaux qui respecte cette singularité et réduire la prolifération des homonymes inutiles, inutiles puisqu'il n'est pas souhaitable de multiplier les entités, est rendu possible par l'analyse proposée dans la Grammaire de Constructions. Les objets en sont les Constructions dont la définition empruntée à Goldberg (1995) est la suivante :

« $\mathrm{C}$ est une CONSTRUCTION ssi $\mathrm{C}$ est une paire forme-sens $<\mathrm{Fi}$, Si> telle qu'un aspect de Si n'est pas strictement prédictible étant donné les composants de $\mathrm{C}$ ou étant donné une Construction préalablement établie. » (p.4)

10 Du point de vue de la syntaxe, le prototype de la Construction, c'est $\mathrm{S}$, puisque la propriété d'assertion de la phrase $\mathrm{S}$ ne découle pas des propriétés de GN ou de GV, qui entrent dans la Construction. Ce qui caractérise donc les Constructions, c'est la non prédictibilité, c'est-à-dire l'arbitraire de l'association de la forme et du sens; toute Construction ainsi définie est une nouveauté, et non le résultat d'une créativité. Les Constructions sont en nombre fini dans une langue.

11 Les morphèmes sont des cas de Constructions « puisqu'on ne peut pas prédire leur sens à partir de leur composition». Le travail d'analyse proposé par la Grammaire de Constructions peut donc s'appliquer à des unités et pas seulement à des syntagmes. La notion de Construction va donc ici s'appliquer à deux niveaux : d'une part on considère 
qui comme une Construction; d'autre part la question se pose de savoir si les différents emplois dans lequel on l'observe sont des Constructions.

\section{Les données}

12 Il va donc s'agir de montrer que le même mot qui appartient à plusieurs Constructions, en commençant par le cas le mieux connu, celui de l'interrogatif, pour ensuite examiner les emplois moins bien répertoriés, à savoir dans l'ordre, le relatif sans antécédent; l'indéterminatif généralisé ; le distributif; et enfin le cas le plus problématique, celui du relatif prépositionnel.

\subsection{Qui interrogatif}

13 L'identification de qui interrogatif est connue, tant dans la procédure à suivre, que dans les résultats (Kayne 1974-75, Godard, 1988). Il s'est agi, en effet, de montrer qu'il y a bien deux mots qui, l'un relatif, l'autre interrogatif, et que ce dernier est distinct de l'interrogatif que. On écrira désormais l'interrogatif, que nous étudions, qui?, pour le distinguer du relatif, noté qui. On se contentera de résumer les résultats obtenus par Kayne et Godard.

14 La première étape de la procédure consiste à constater les propriétés suivantes, qui sont distinctives.

Qui relatif est seulement sujet, et il n'est pas sensible aux traits sémantiques de son antécédent : c'est un pronom anaphorique qui peut avoir pour antécédent n'importe quel GN, à tête dotée du trait Humain ou inanimé indifféremment. D'autre part qui relatif entre avec que relatif dans une relation de déclinaison, puisque que est seulement complément d'un verbe (ou attribut) et qu'il partage toutes les autres propriétés avec qui ; il s'agit d'un pronom anaphorique.

Qui? interrogatif se distingue de qui relatif, d'une part parce qu'il remplit toutes les fonctions, sujet, complément d'un verbe (ou attribut), complément d'une préposition dans un groupe prépositionnel; d'autre part parce qu'il a une interprétation sémantique inhérente, puisqu'à une question en qui? on ne peut répondre naturellement que par l'emploi d'un GN dont la tête est un nom Humain. Mais il se distingue aussi de que interrogatif, par l'interprétation sémantique inhérente: que pose une question dont la réponse attendue doit concerner un GN dont la tête est un nom inanimé ; de plus, alors qu'en ancien français que? est disponible pour les fonctions sujet et complément, il n'est plus acceptable en français moderne que pour la fonction complément de verbe, et la fonction attribut ${ }^{2}$.

17 Une dernière propriété permet de distinguer qui? de qui : qui ? a les propriétés d'un GN, alors que qui n'en est pas un, comme l'a montré Kayne (1974 \& 1975) à qui nous renvoyons. Or qui ? apparaît non seulement dans la position COMP comme le relatif, mais également en position argumentale, puisque les questions directes ont en français deux formes :

5. Qui as-tu vu? 
6. Tu as vu qui? S'il est vrai que les formes avec ce qui / ce que sont «sans antécédent » du point de vue sémantique, puisque ce est dénué d'interprétation, il y a bel et bien un antécédent du point de vue de la syntaxe, et cet antécédent est $c e$.

$$
\begin{array}{|l|l|}
\hline \text { 7. Qui m'aime me suive. } \\
\hline & \text { 8. Embrassez qui vous voudrez. } \\
\hline & \text { 9. Je parle à qui me plaît. } \\
\hline & \text { 10. N'est pas gaulliste qui veut. } \\
\hline
\end{array}
$$

22 La différence entre les relatives en qui et les relatives en ce qui / ce que est la suivante: alors que qui peut être aussi bien sujet (7), (9) et (10) que complément de verbe (8) ou de préposition, l'alternance qui / que dans les formes ce qui / ce que atteste qu'il s'agit alors des formes du relatif qui se déclinent en nominatif/ accusatif :

11. J'ai trouvé ce qui convient. 
12. J'ai trouvé ce que vous cherchez.

vu de ces exemples que la forme qui telle qu' elle apparaît dans (7) à (10) a de nombreuses propriétés communes avec la forme qui ?: il s'agit d'un GN, comme l'attestent les fonctions remplies; dont l'interprétation est comme pour ce dernier " Humain » : la seule différence tient à ce qu'il n'y a plus d'interprétation «Interrogatif », mais une interprétation "Indéfini », qu'on peut paraphraser par "n'importe qui ». Si on arrive à montrer que cette interprétation n'est pas incompatible avec celle d'interrogatif, et que les deux interprétations dépendent des Constructions dans lesquelles le mot entre, l'identification sera justifiée. On pourra dire alors que les relatives sans antécédent sont une construction dans laquelle entre qui ?

\subsection{Qui ? indéterminatif généralisé}

La terminologie proposée par Tesnière (1959, chap.265) permet d'identifier clairement cet emploi, classé par les grammaires soit dans les concessives (Sandfeld, 1936), soit dans les relatives (Riegel et al. 1994). Sandfeld présente les formes de la façon suivante :

«...une proposition relative indépendante se change en proposition concessive par l'adjonction de que au conjonctif ("Qui que tu sois, ange ou démon, ne te fais pas attendre plus longtemps). »(\$235)

Riegel et al. considèrent ces formes comme des « relatives compléments circonstanciels de concession »:

«(elles) sont introduites par qui que, quoi que, où que suivis du subjonctif: le premier élément peut s'interpréter comme un pronom indéfini caractérisant une catégorie sémantique (animé, inanimé, lieu), le second est le relatif qui lui assure une place et une fonction dans la subordonnée de sens concessif : "Qui que ce soit, c'est un malin. » (p.488-489).

Alors que Sandfeld rapproche qui du relatif sans antécédent, Riegel et al. proposent une nouvelle catégorie, celle de l'indéfini. On peut noter qu'ici intervient pour la première fois le terme " pronom indéfini " pour identifier qui. Cette identification est importante : elle permet de rappeler que du point de vue de l'étymologie, la forme qui ? se rattache au thème indo-européen ${ }^{*} q w^{-}$, qui entre dans la formation des indéfinis et des interrogatifs en latin et en grec (Frei, 1940). Le fait que l'interrogatif soit un indéfini est donc un phénomène établi par d'autres traces que l'organisation synchronique du système.

Or la forme qui de l'indéterminative généralisée a les mêmes propriétés que celles déjà établies pour l'interrogatif et le relatif sans antécédent: c'est un $\mathrm{GN}$ - il a toutes les fonctions du GN - et il a l'interprétation « Humain » caractéristique de ce pronom.

13. Avec qui que ce soit que vous partiez, prenez votre temps.

\subsection{Qui ? distributif}

Sous ce nom, Riegel \& al.(1994, p. 212), de même que Grevisse, rendent compte de l'emploi suivant de qui : 
14. Ils lui ont offert qui un livre, qui des fleurs (Riegel et al.,1994, p. 212).

15. L'auditoire gémit, en voyant, dans l'enfer tout ouvert, qui son père et qui sa mère, qui sa grandmère et qui sa sœur. (A.Daudet, in Grevisse, §542).

On peut décrire les propriétés de cette Construction comme suit :

- Qui est obligatoirement répété, au moins deux fois.

16. Pour être lauréats, ils devaient avoir fait, dans un temps donné, qui sculpteur, le modèle en terre glaise d'une statue; qui peintre, l'un des tableaux que vous pouvez voir à l'école des Beaux-Arts; qui musicien, une cantate; qui architecte, un projet de monument. (Balzac, La Rabouilleuse).

- Qui est apposé à un GN doté d'une fonction dans la phrase ou à un pronom clitique sujet ou objet :

17. Dix personnes sont déjà venues me prier de lui proposer, qui un fils, qui un frère, qui un ami.

18. Aux moujiks accourus ils distribuent à qui une jambe, à qui un bras. (Sandfeld, 1928, \$239bis)3.

19. Ils achetèrent vingt francs, qui un tableau, qui une gravure.

20. Je les regardais, buvant qui une bière, qui un verre de vin.

21. Je les voyais lire qui un livre, qui une revue.

- Qui est apposé à un GN doté du trait [+Humain] :

\begin{tabular}{|l|l|l|}
\hline & 22. & a. Les livres sont éparpillés les uns sur les étagères, les autres sur le sol. \\
\hline & & b. ${ }^{*}$ Les livres sont éparpillés qui sur les étagères, qui sur le sol \\
\hline
\end{tabular}

- Qui est suivi soit d'un complément obligatoire du verbe (14), (15), soit d'un complément non argumental, un ajout (26), soit des deux (23).

23. Les dames et les jeunes filles en villégiature venaient [à la gare] attendre, qui un père dans les rubans, qui un frère dans les blés, qui un époux dans les pierres précieuses. (Sandfeld, 1928, §239bis)

- Le terme, auquel est apposé qui, est soit un GN pluriel (19), (17), soit un nom qui désigne une collectivité (15), (24), soit un distributif comme chaque (25), chacun (26):

24. La foule applaudissait qui le bateleur, qui le prestidigitateur.

25. Chaque habitué apportait qui sa canne, qui son manteau, qui sa lanterne. (Balzac, La Vieille fille) 
26. Chacun partait qui avec un bouquet, qui avec un cadeau.

27. Quatre d'entre elles - Lola, Manette et Cora, et la chatte Nonoche, ont parlé déjà, qui dans "les Vrilles de la Vigne », qui dans "L'Envers du music-hall. »

28. Ils vont l'un à Paris, l'autre à Lyon.

Deux différences apparaissent: la première est que l'un... l'autre peut apparaître en position argumentale. Ainsi les deux GN peuvent être sujets :

29. L'une chante, l'autre pas. (Milner 1984)

30. L'une chante, l'autre danse.

Deuxième différence, sémantique, celle-là : là où l'un ...l'autre énumère exhaustivement les éléments d'un ensemble, qui...qui énumère sans que l'énumération soit exhaustive un ensemble dont dès lors la relation entre les éléments distribués reste inconnue.

On peut donc au vu de ce jeu de ressemblances et de différences envisager d'attribuer à qui dans la Construction distributive la combinaison suivante de traits, combinaison paradoxale si l'on veut: anaphorique et indéfini. Un deuxième résultat important est le suivant : le trait indéfini de qui se distingue de celui de quelqu'un aussi bien que de celui de chaque, qui est distributif comme qui, mais alors que chaque a la propriété de faire l'énumération exhaustive des éléments d'un ensemble, qui fait une distribution aléatoire sur un ensemble. Les traits constants qui justifient l'assimilation de qui distributif à qui interrogatif sont la catégorie [GN], et le trait [Humain], qui restent constants.

\subsection{Qui ? relatif prépositionnel}

La dernière occurrence est la plus inattendue : c'est celle du relatif prépositionnel : 
a. Le livre auquel je fais référence / l'auteur auquel je fais référence.

b. *le livre à qui je fais référence / l'auteur à qui je fais référence. opposition entre qui et lequel (amalgamé avec à sous la forme auquel) : qui est réservé à un antécédent [+Humain], alors que auquel manifeste la même indistinction que la paire du relatif que / qui par rapport à son antécédent. Cette occurrence de qui est particulièrement inattendue parce qu'elle répond aux propriétés suivantes : d'une part il s'agit d'un relatif, donc d'un anaphorique, d'autre part cet anaphorique impose à son antécédent d'avoir le trait [+Humain], ce qui constitue un paradoxe, si on compare un tel anaphorique aussi bien au relatif qui / que qu'au pronom il, lesquels n'imposent pas de condition sur leur antécédent nominal.

Pour éclairer ce phénomène, on peut le rapprocher de phénomènes semblables qui mettent en cause des GP comportant des pronoms.

\subsubsection{Propriétés des pronoms dans les GP}

On peut en effet envisager une sorte de régularisation du phénomène si on fait l'observation suivante : les pronoms personnels anaphoriques dominés par GP ont le trait [+Humain]; ce sont des formes fortes et ils contrastent en cela avec un autre pronom clitique qui appartient à la série anaphorique et qui, lui, est disponible pour un antécédent indifféremment [+Humain] ou [-Humain]. On distingue ainsi, lorsqu'un pronom anaphorique est concerné, deux formes de GP : l'un est un pronom [pro GP] clitique, l'autre est un constituant GP normal analysable en $\mathrm{P}+\mathrm{GN}$, où le GN a la forme d'un pronom fort (Delaveau \& Kerleroux, 1985). C'est ce dernier pronom, lui (forme forte), qui a le trait [+Humain].

\begin{tabular}{|l|l|l|}
\hline & 32 & a. J'en ai parlé, de vous. \\
\hline & & b. J'en ai besoin, de ce livre. \\
\hline & & c. Paul, j'ai parlé de lui. \\
\hline & & d. ${ }^{*} C e$ livre, j'ai parlé de lui. \\
\hline & 33 & a. J'y pense, à vous. \\
\hline & b. J'y vais, à ce congrès. \\
\hline & c. Paul, je pense à lui. \\
\hline & d. *Ce congrès, je pense à lui. \\
\hline
\end{tabular}

39 On voit que l'occurrence des GP [à/de lui] n'est possible que si l'antécédent est [+Humain] Par contre, l'occurrence des pronoms GP en et y est possible quel que soit l'antécédent, en 
particulier dans la Construction de la dislocation droite (Delaveau \& Kerleroux, 1985). C'est la même contrainte qui s'applique dans le contraste à qui/auquel : à qui n'est possible que si l'antécédent est [+Humain], auquel est indifférent à son antécédent.

\subsubsection{Les prépositions orphelines}

On observe encore ce contraste entre des GP dans le cas des prépositions orphelines, sous la forme d'une distribution complémentaire : le pronom fort lui / elle est obligatoire si l'antécédent est [+Humain], la préposition est orpheline si l'antécédent est [- Animé]:

\begin{tabular}{|l|l|l|}
\hline & 34 & a. Cette valise, je voyage toujours avec. \\
\hline & & b. *Marie, j'aime voyager avec. \\
\hline & & c. Marie, j'aime voyager avec elle. \\
\hline 35 & a. L'entracte, Paul est parti avant. \\
\hline & & b. ${ }^{*}$ Marie, Paul est parti avant. \\
\hline & & c. Marie, Paul est parti avant elle. \\
\hline
\end{tabular}

L'existence d'une distinction entre un pronom spécialisé pour un antécédent [+Humain] et un pronom non spécialisé semble donc attesté pour un ensemble de GP: ceux qui mettent en cause le relatif (à qui / auquel), ceux qui mettent en cause l'anaphorique (de lui / en et à lui / y) et enfin le système qui met en cause le contraste entre une préposition + pronom anaphorique fort et une préposition seule dans le cas des préposition pleines comme avec, sans, devant, derrière.

Or dans le cas des pronoms anaphoriques non relatifs, l'alternance est assurée par un matériel grammatical disponible - pronoms forts vs pronoms clitiques, ou pronoms forts vs absence de pronom. Dans le cas du relatif, la forme qui est entièrement vouée au cas nominatif: la supplétion se fait par l'usage du pronom qui? dont l'interprétation est [Humain] et qui sature ainsi le contraste avec le relatif auquel.

L'analyse proposée confirme la conclusion de Kayne selon laquelle qui relatif n'est pas un GN, pas plus que le relatif que. Si qui relatif était un GN, il n'y aurait pas d'obstacle à ce qu'il entre dans la Construction $\mathrm{P}+\mathrm{GN}$. Mais s'il y entrait, la contrainte sur l'antécédent [Humain] que l'on observe ne devrait pas exister puisque le nominatif qui admet toute espèce d'antécédent. La répartition des formes est donc étayée par là aussi. Par ailleurs, le raisonnement est confirmé par l'existence dans le cas du relatif prépositionnel de la forme quoi (qui est également celle de l'interrogatif) qui est employé en français contemporain dans le contexte restreint d'un antécédent comme cela ou la chose :

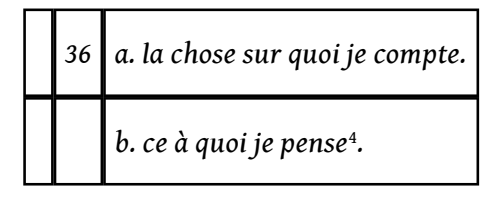



(1995) formule :

«Si deux Constructions sont syntaxiquement distinctes, alors elles doivent être sémantiquement ou pragmatiquement distinctes ». (p.67) signification, ce qui est le cas du mot qui ?, qui est donc polysémique. S'agissant de syntagmes, on peut chercher les corrélations de cette polysémie du côté du lexique; s'agissant de mots grammaticaux comme qui?, on peut les chercher du côté des Constructions dans lesquelles ils entrent : c'est le principe qui a guidé la présentation des données. Si les Constructions syntagmatiques tirent leur polysémie des éléments constituants, les mots grammaticaux tirent leur polysémie de leur participation à des Constructions syntagmatiques.

Cette polysémie est organisable autour d'un « sens central » : cette notion essentielle est opposée par Goldberg à celle de "sens abstrait ", c'est-à-dire à celle d'un sens minimal qui serait commun à l'ensemble des occurrences. Le « sens central » est aussi complet que n'importe laquelle des variations observées; il est justifié par le fait que la Construction à laquelle il est attribué paraît la moins marquée, la moins soumise à des contraintes de distribution interne ou externe. 

ci organise l'ensemble des Constructions concernées en un réseau où les héritages se font d'une part à partir du sens central, d'autre part à partir d'autres Constructions qui sont activées pour rendre compte d'une interprétation. Il y a héritage chaque fois que deux Constructions distinctes présentent une propriété commune, qui est attribuable à une troisième Construction laquelle motive cette propriété commune : la relation d'héritage est représentée par des liens (figurés dans le réseau des Constructions par une flèche dans la figure 1). la Construction GP ; c'est cette multiplicité de liens qui permet de représenter le caractère paradoxal de cet emploi où qui ? perd le trait [Indéfini] et acquiert le trait [Anaphorique]. Le dernier emploi, celui du distributif, a à la fois le trait [Indéfini] et le trait [Anaphorique]. Il n'hérite que du sens central, dans la mesure où il ne manifeste que les propriétés de GN par son emploi en apposition. Et c'est cet emploi d'apposition qui, par son interprétation prédicative, lui donne le trait Anaphorique.

\section{L'interprétation de Qui ?}

Reste à caractériser de façon plus précise le caractère indéfini de qui ? Si un mot grammatical est singulier, il l'est par son interprétation: dans l'ensemble des indéfinis, quelle est la spécificité de qui ?

Les questions partielles, qui portent sur un constituant de la phrase, contiennent un mot qu- comme qui ? Elles sont également appelées questions ouvertes, par opposition aux questions totales dites questions fermées. Kuroda (1969), reprenant une proposition de Harris, envisage qu'une question avec un mot qu-, par exemple "Qui a acheté ces livres? » ait pour structure profonde une disjonction infinie de constituants. Il écarte cette solution de la syntaxe, étant donné que celle-ci ne peut proposer que des règles finies. Mais si on déplace la disjonction infinie de l'ordre de la syntaxe à celui de la 
sémantique, l'interprétation de qui ? comme une disjonction infinie permet de rendre compte d'une part de l'interprétation de l'interrogatif, d'autre part de la spécificité de qui ? parmi les indéfinis.

Une interrogation comme « Tu as vu qui ? " peut en effet être interprétée comme « Tu as vu x ou y ou z, etc.». Qui ? est donc interprété comme la disjonction infinie de GN dotés du trait [Humain].

61 D'autre part, l'emploi distributif de qui ? a permis de contraster « qui.. qui » avec « l'un... l'autre »: alors que le calcul de l'addition faite par «l'un... l'autre » peut être effectué, ce qui donne pour résultat un ensemble fini d'éléments, l'ensemble sur lequel qui ? effectue un prélèvement est un ensemble infini. Ceci permet d'expliquer que l'énumération de qui ? dans l'emploi distributif ne permet pas d'opérer la distribution des éléments mis en relation entre le terme relaté, l'apposition qui ?, et les compléments ou les ajouts de la Construction.

Figure 1 : RESEAU des LIENS de QUI ?

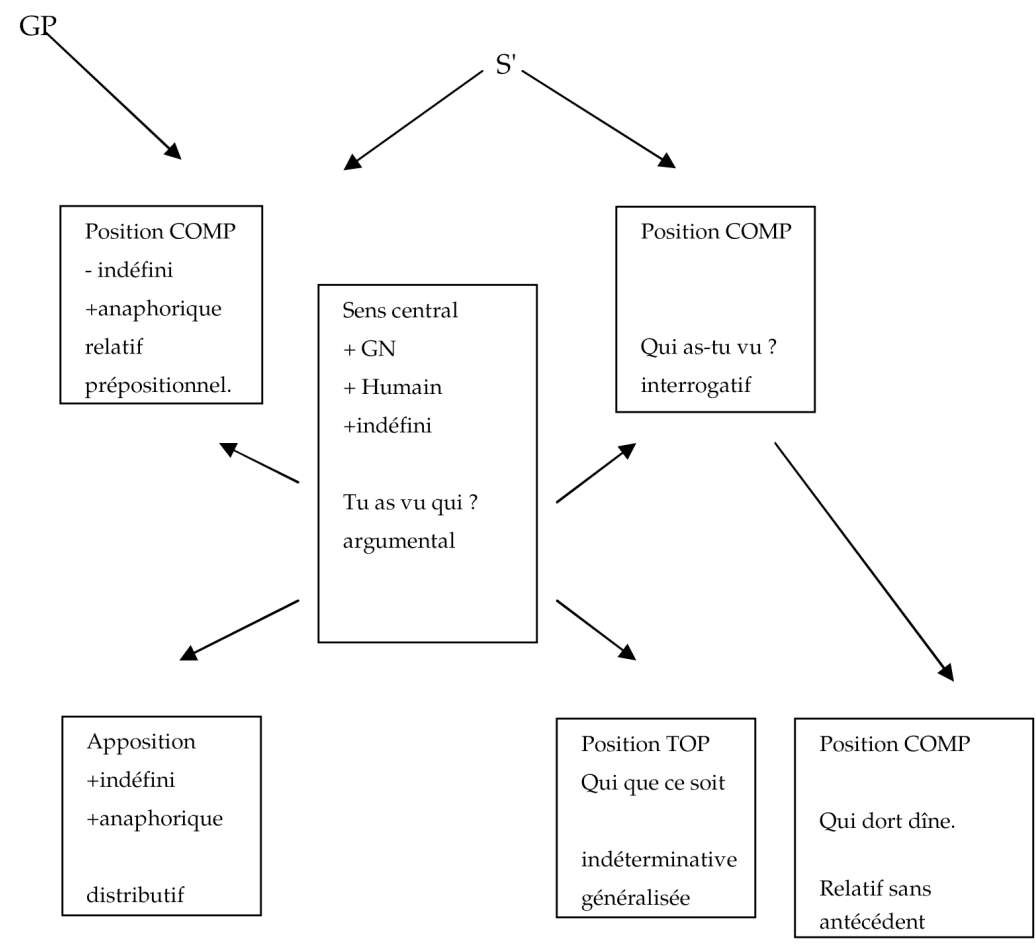

\section{Conclusion}

Les propositions de la Grammaire de Constructions permettent de répondre à l'hypothèse formulée par Emonds de la singularité des mots grammaticaux. Le traitement évite la prolifération d'homonymes, au profit d'une description de la polysémie de qui ?. Cette polysémie est sous le contrôle des distributions dans lesquelles apparaît le mot. Ces distributions sont purement et simplement stipulées - c'est le cas du sens central proposé ainsi que du sens distributif où l'on constate que qui ? est un GN, et peut en tant que tel avoir toutes les fonctions de GN, entre autres celles d'apposition, avec des contraintes 
particulières - celle de la répétition. Ou bien ces distributions sont sous le contrôle de Constructions spécifiées, comme S' ou comme GP.

Le réseau des liens est un constat : il enregistre les phénomènes dans leur hétérogénéité. Les héritages sont constatés. Une valeur explicative peut être accordée au fait qu'il faille plusieurs héritages pour rendre compte de l'emploi marqué, celui du relatif prépositionnel.

\section{BIBLIOGRAPHIE}

DELAVEAU, A. \& KERLEROUX, F. (1985) Problèmes et exercices de syntaxe du français, Colin, Paris.

EMONDS, J. (1985) A Unified Theory of Syntactic Categories, Foris, Dordrecht.

FREI, H. (1940) Interrogatif et indéfini, Geuthner, Paris.

GODARD, D. (1988) La syntaxe des relatives en français, éd. du CNRS, Paris.

GOLDBERG, A. (1995) A Construction Grammar Approach to Argument Structure, The University of Chicago Press, Chicago.

KAYNE, R. (1974 \& 1975) « French relative que », Recherches Linguistiques, Paris VIII, Vincennes II, p. 40-61, \& III, p. 27-92.

KURODA, S.Y. (1969) «Les transformations d'attachement », Aux quatre coins de la linguistique, 1979, Le Seuil, p. 43-76.

MILNER, J.C. (1984) «Syntaxe et sémantique du constituant réciproque l'un...l'autre », Recherches sur l'anaphore, U.R.A. 1028, Université de Paris 7, p. 35-67.

RIEGEL, M., PELLAT, J.C., RIOUL, R. (1994) Grammaire méthodique du français, PUF, Paris.

SANDFELD, Kr. (1928) Syntaxe du français contemporain. Les pronoms. Champion, Paris.

SANDFELD, Kr. (1936) Syntaxe du français contemporain. Les propositions subordonnées. Droz, Genève.

TESNIERE, L. (1959) Eléments de syntaxe structurale, Klincksieck, Paris.

ZWICKY, A. (1994) « Dealing out meaning: Fundamentals of syntactic Constructions.' Proceedings of the 20th annual meeting of Berkeley Linguistic Society.: p. 611-625.

\section{NOTES}

1. Le terme est emprunté à Tesnière (1959, chap.265).

2. Une autre propriété de que interrogatif est qu'il est clitique : il ne peut être séparé du verbe dont il est complément.

3. On peut hésiter quant à la grammaticalité de cet exemple. Il n'est présent qu'à titre de donnée attestée, quelles que soient les modalités de sa fabrication.

4. On peut signaler - ce qui soutient l'analyse des interrogatifs comme des indéfinis - l'usage dans le français du Nord des formes : «Alors tu me diras quoi. » et «Je te dirai quoi. », qui pour 
conclure un échange, signifient « tu me diras ça ou ça que tu vas trouver». On voit mal comment on pourrait dire autrement cela.

\section{RÉSUMÉS}

L'analyse classique des mots grammaticaux revient à les identifier par l'appartenance de classe qui regroupe plusieurs unités sur la base d'une ou deux propriétés partagées, ainsi des pronoms interrogatifs ou des pronoms relatifs. Il s'agit de montrer qu'un autre point de vue est possible : celui énoncé par J.Emonds et rendu opératoire par la grammaire de Constructions, qui considère chaque mot grammatical comme un individu spécifié par un ensemble de combinatoires et par une interprétation sémantique «centrale ». L'application de cette hypothèse à qui ? - celui qui est identifié comme interrogatif par opposition au relatif - rassemble sous une seule unité un ensemble d'occurrences distinguées par les grammaires : l'interrogatif, le relatif sans antécédent, l'indéterminatif généralisé, le distributif, et le relatif prépositionnel. L'attribut «indéfini » est justifié par l'hypothèse que le sens central de qui ? est une disjonction infinie de GN [Humain].

Classical analysis of grammatical words identifies them through their class which puts them together on the basis of one or two shared properties, for example interrogative pronouns ou relative pronouns. The article shows that an other point of view is possible, one which has been proposed by J.Emonds and can be developped by Construction Grammar. In this theory, every grammatical word is an individuate specified by a set of distributions and a "central » semantic interpretation. Studying qui? - the interrogative pronoun - within such an hypothesis conducts to unify under a single item a set of occurences which grammars distinguish: interrogative, free relative, concessive, distributive and prepositional relative. The «indefinite " attribute is justified by the consideration that the central meaning of qui? is an infinite disjonction of [Human] NP.

\section{AUTEUR}

\section{ANNIE DELAVEAU}

Université de Paris X, EA 672 